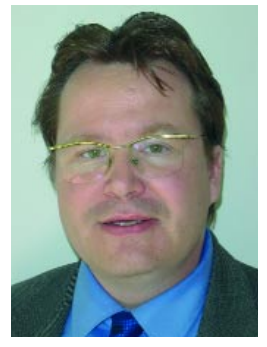

Georg Juckel

\title{
Früherkennung beginnender schizophrener Erkrankungen
}

\author{
Georg Juckel', Frauke Schultze-Lutter², Stephan Ruhrmann²
}

${ }^{1}$ Früherkennungs- und Therapiezentrum (FETZ) für beginnende Psychosen Berlin-Brandenburg, Klinik für Psychiatrie und Psychotherapie, Charité, Campus Mitte, Berlin

2Früherkennungs- und Therapiezentrum (FETZ) für psychische Krisen, Klinik für Psychiatrie und Psychotherapie, Universität zu Köln

psychoneuro 2003; 30 (3): 153-159

Schizophrene Störungen haben nach wie vor eine schlechte Prognose. Chronische Verläufe mit deutlichen sozialen Einbußen und progressiven neurobiologischen Veränderungen sind häufig. Heute weiß man, je länger die unbehandelte Phase vor Ersthospitalisierung andauert, desto schlechter ist die spätere Prognose. Daher sind Strategien der Früherkennung von Patienten im Prodromalzustand sinnvoll, um diese Phase bereits therapeutisch zu nutzen. Zur Erkennung insbesondere früher Prodromalstadien hat sich das Bonn-Kölner Basissymptomkonzept, insbesondere eine Untergruppe von kognitiv-perzeptiven Basissymptomen, als spezifisch und prädiktiv hinsichtlich der Übergangswahrscheinlichkeit in das Vollbild einer schizophrenen Psychose herausgestellt. Bei den späteren Abschnitten des Prodroms, zu dem ein Übergang in eine manifeste Psychose bereits nahe bevor steht, werden den Kriterien einer schizotypischen Persönlichkeitsstörung entlehnte sog. attentuierte psychotische Symptome (APS) und brief limited intermittent psychotic symptoms (BLIPS) herangezogen. Eine Vielzahl strukturierter Interviews und Ratingskalen (z.B. SIPS, SPIA, ERIraos) ist entwickelt worden, um Früherkennung schizophrener Prodromalzustände standardisiert durchzuführen. Diese sind eingebettet in weitere Untersuchungen z.B. neuropsychologische, neurophysiologische und Bildgebungsuntersuchungen. Eine erfolgreiche Früherkennung setzt jedoch eine möglichst breit wirksame Sensibilisierung der allgemeinen und Fachöffentlichkeit für das Thema der Schizophrenie und ihres Frühverlaufs voraus. Die Vernetzung von spezialisierten Früherkennungszentren mit möglichst vielen Berufsgruppen, die mit Jugendlichen und jungen Erwachsenen arbeiten, scheint hierbei besonders wichtig zu sein.

tig arbeiten, viele sind berentet, haben keine fertige Ausbildung, Partnerschaft oder Familie. 10\% der Patienten versterben an Suizid in den ersten zehn Jahren ihrer Erkrankung. Die Global Burden of Disease Study der WHO (17) zeigte, dass die Schizophrenie eine Erkrankung ist, die am fünfthäufigsten zur dauerhaften Behinderung führt, noch vor Volkskrankheiten wie den kardiovaskulären Erkrankungen oder Diabetes. Insbesondere die soziale Ver-

sorgung schizophrener Patienten sorgt für hohe Kosten; ca. zehn Milliarden Euro werden jährlich in der Bundesrepublik direkt und indirekt für die Schizophrenie ausgegeben.

Untersuchungen zum Frühverlauf schizophrener Psychosen wie die retrospektive, epidemiologisch ausgerichtete AgeBeginningCourse $(A B C)$-Studie von Häfner und Mitar- beitern (5) konnten zeigen, dass dem Höhepunkt der ersten psychotischen Episode und dem ersten stationären Behandlungskontakt im Mittel ein Zeitraum von über einem Jahr vorangeht, in dem bereits deutliche psychotische Symptome vorhanden sind (Abb.1). Die Dauer dieser so genannten psychotischen Vorphase oder Dauer der unbehandel- 


\section{Tab.1. Folgen einer verzögerten Behandlung}

Ein verzögerter Behandlungsbeginn geht einher

mit:

- einer verzögerten und unvollständigeren Remission der Symptomatik

- längerer stationärer Aufenthaltsdauer und einem höheren Rückfallrisiko

- ausgeprägterer Negativsymptomatik

- einer geringeren Compliance

- einer höheren Belastung der Familie

- einem erhöhten Depressions- und Suizidrisiko

- größeren Belastungen der Arbeits- und Ausbildungssituation

- einem erhöhten Substanzmissbrauch und delinquentem Verhalten

- deutlich höheren Behandlungskosten

ten Psychose (DUP; Duration of Untreated Psychosis) ist mit einem schlechteren klinischen und sozialen Verlauf assoziiert ist (Tab. 1). Ein erstes Ziel war es darum, die Dauer der unbehandelten Psychose durch eine rechtzeitigere Identifikation der Erkrankungsfälle zu verkürzen. So konnte das norwegische TIPSProjekt diese Phase durch intensive Öffentlichkeitsarbeit und Einsatz mobiler psychiatrischer Teams um im Schnitt fast ein halbes Jahr verkürzen (11).

Um die Dauer der unbehandelten Psychose weiter verkürzen zu können, richtete sich das Interesse im Weiteren auf die Vorhersage der psychotischen Erstmanifestation. Auch hierzu hatte die ABC-Studie wichtige Befunde geliefert, da sie hatte zeigen können, dass in drei Viertel der untersuchten Fälle der Manifestation einer Psychose eine Prodromalphase von fünf bis sechs Jahren voranging. Ausgehend von den retrospektiven Beschreibungen wurden im angloamerikanischen Raum Kriterien entwickelt, die auf eine Früherkennung im fortgeschrittenen Stadium der Prodromalphase, nahe am Übergang zur Psychose zielen (23). Parallel hierzu gibt es in Deutschland eine lange, auf Gerd Huber (7) zurückgehende Tradition der Früherkennung, die sich auf das Konzept der Basissymptome stützte.

Wesentlich verstärkt wurde das Interesse an einer Früherkennung in der Prodromalphase noch durch jüngste Ergebnisse, die die Hypothese zulassen, dass die Entwicklung einer Psychose mit einem progredienten hirnmorphologischen Defizit verbunden ist. So konnten Pantelis et al. (18) im Längsschnitt zeigen, dass mit der Erstmanifestation einer schizophrenen Psychose eine Reduktion der grauen Substanz links parahippokampal, fusiform, orbitofrontal, zerebellär und im Cingulum assoziiert war (Abb. 2). Dazu passend fanden Geschwandtner et al. (3), dass bereits vor Ausbruch der Psychose, also im sog. Prodromalzustand, deutliche Veränderungen in der Neuropsychologie (vor allem in Tests für den Frontallappen), in der Feinmotorik und in den Augenfolgebewegungen, ähnlich den Patienten im Vollbild einer schizophrenen Psychose, vorhanden sind.

\section{Prodromalsymptome psychotischer Störungen}

Das Prodrom ist durch eine vielfältige, oftmals unspezifische Symptomatik gekennzeichnet (22). Die teils prominente Schilderung von unspezifischen Beschwerden wie etwa

- Konzentrationsstörungen

- Antriebsarmut

\section{Tab. 2 Basissymptome mit hinlänglicher* Häufigkeit}

\begin{tabular}{|c|c|c|c|c|c|c|}
\hline BSABS-Prodromalsymptom & Sensitivität & Spezifität & $\begin{array}{l}\text { Positive } \\
\text { prädiktive } \\
\text { Stärke }\end{array}$ & $\begin{array}{l}\text { Negative } \\
\text { prädiktive } \\
\text { Stärke }\end{array}$ & $\begin{array}{l}\text { Falsch- } \\
\text { positive } \\
\text { Vorhersagen }\end{array}$ & $\begin{array}{l}\text { Falsch- } \\
\text { negative } \\
\text { Vorhersagen }\end{array}$ \\
\hline Gedankeninterferenz & 0,42 & 0,91 & 0,83 & 0,62 & $4,4 \%$ & $28,8 \%$ \\
\hline $\begin{array}{l}\text { Zwangähnliches Perseverieren } \\
\text { bestimmter Gedanken }\end{array}$ & 0,32 & 0,88 & 0,71 & 0,57 & $6,3 \%$ & $33,8 \%$ \\
\hline Gedankendrängen, -jagen & 0,38 & 0,96 & 0,91 & 0,62 & $1,9 \%$ & $30,6 \%$ \\
\hline $\begin{array}{l}\text { Blockierung des jeweiligen } \\
\text { Gedankenganges }\end{array}$ & 0,34 & 0,86 & 0,71 & 0,57 & $6,9 \%$ & $32,5 \%$ \\
\hline Störung der rezeptiven Sprache & 0,39 & 0,91 & 0,82 & 0,61 & $4,4 \%$ & $30,0 \%$ \\
\hline $\begin{array}{l}\text { Störung der Diskriminierung } \\
\text { von Vorstellungen und Wahr- } \\
\text { nehmungen bzw. Phantasie- } \\
\text { und Erinnerungsvorstellungen }\end{array}$ & 0,27 & 0,95 & 0,84 & 0,57 & $2,5 \%$ & $36,3 \%$ \\
\hline $\begin{array}{l}\text { Eigenbeziehungstendenz, } \\
\text { „Subjekt-Zentrismus“ }\end{array}$ & 0,39 & 0,89 & 0,78 & 0,60 & $5,6 \%$ & $30,0 \%$ \\
\hline Derealisation & 0,28 & 0,90 & 0,73 & 0,56 & $5,0 \%$ & $35,6 \%$ \\
\hline $\begin{array}{l}\text { Optische Wahrnehmungs- } \\
\text { veränderungen }\end{array}$ & 0,46 & 0,85 & 0,75 & 0,62 & $7,5 \%$ & $26,9 \%$ \\
\hline $\begin{array}{l}\text { Akustische Wahrnehmungs- } \\
\text { veränderungen }\end{array}$ & 0,29 & 0,89 & 0,72 & 0,53 & $5,6 \%$ & $35,0 \%$ \\
\hline
\end{tabular}


- gedrückte Stimmung

- Interesselosigkeit

- $\quad$ sozialer Rückzug

- Gefühlsschwankungen

- Schlaf- und Appetitstörungen

- Leistungsabfall

wurde lange als Argument gegen eine mögliche Früherkennung ins Feld geführt, da solche Symptome bei Jugendlichen und jungen Erwachsenen auch z.B. im Rahmen einer Adoleszentenkrise, einer beginnenden Depression oder Persönlichkeitsstörung auftreten können. Die im amerikanischen DSM-III und DSM-III-R unternommene Operationalisierung von prodromalen bzw. residualen Symptomen wurde darum aufgrund mangelnder Reliabilität und unklarer Validität im DSM-IV wieder verlassen (9). Stattdessen wurde gefordert, dass vor allem mit Hilfe prospektiver Langzeituntersuchungen geklärt werden müsse, ob alternative Kriterien oder Konzepte eine ausreichende prädiktive Validität besitzen, bevor sie erneut Eingang in die klinische Diagnostik finden.

Einen alternativen Ansatz stellt das von Huber in den 60er-Jahren entwickelte Basissymptomkonzept dar. Es hat seinen Ursprung in der Beobachtung von Defizienzen, die bereits langjährig vor der ersten akuten Episode und auch im weiteren Verlauf auftreten, von den Betroffenen selbst wahrgenommen und berichtet werden (8). Basissymptome sind milde, meist subklinische, aber nichtsdestotrotz häufig starken Beschwerdedruck verursachende Selbstwahrnehmungen von Störungen des Antriebes, des Affekts, von Denk- und Sprachprozessen, der Wahrnehmung, der Propriozeption und der Motorik.

Klosterkötter und Mitarbeiter (10) konnten nun zeigen, dass vor allem eine Untergruppe von kognitivperzeptiven Basissymptomen prädiktiv hinsichtlich der Übergangswahrscheinlichkeit in das Vollbild einer schizophrenen Psychose ist (s. Tab. 2). Sie untersuchten nach einem Katamnesezeitraum von im Durchschnitt zehn Jahren 160 Patienten nach, die sich wegen unklarer psychischer Beschwerden in einer psychiatrischen Spezialambulanz vor- gestellt hatten. Diejenigen, die in der Erstuntersuchung mindestens eines der Basissymptome aufgewiesen hatten, waren nachfolgend überdurchschnittlich häufiger an einer Schizophrenie erkrankt als diejenigen Patienten ohne solche Beschwerdeschilderungen.

Basissymptome können bereits in frühen Abschnitten des initialen Prodroms auftreten. Sie haben somit den Vorteil, Prodromalzustände auch schon zu einem Zeitpunkt erfassen zu können, zu dem Funktionseinbußen etwa im sozialen und beruflichen Bereich noch nicht oder erst in geringem Ausmaß aufgetreten sind. Dies gilt auch für die prädiktiven Denkstörungen, die zusammen mit Konzentrationseinbußen in der ABC-Studie von 16\% der Patienten als eines der ersten Symptome berichtet wurden.

Demgegenüber zielen die im angloamerikanischen Raum konzipierten Prodromalkriterien explizit auf einen späteren Abschnitt des initialen Prodroms, zu dem ein Übergang in eine manifeste Psychose bereits nahe bevor steht. Hierbei werden v.a. den DSM-IVKriterien einer schizotypen Persönlichkeitsstörung entlehnte attentuierte psychotische Symptome (APS) und sog. brief limited intermittent psychotic symptoms (BLIPS) herangezogen (Tab. 3 ).

In Prodromalzuständen kann die differentialdiagnostische Abgrenzung gegen eine drogenassoziierte Symptomatik im Rahmen eines Ge-

\section{Tab. 3 Kriterien des „Ultra-high risk“ Ansatzes der Früherkennung}

Vorliegen mindestens eines der folgenden attenuierten psychotischen Symptome, mehrfach über mindestens eine Woche auftretend

- Beziehungsideen

- Eigentümliche Vorstellungen oder magisches Denken

- Ungewöhnliche Wahrnehmungserlebnisse

- Eigenartige Denk- und Sprechweise

- Paranoide Ideen

Vorliegen mindestens eines der folgenden kurzfristigen psychotischen Symptome (BLIPS) mit einem Auftreten von weniger als sieben Tagen und nicht häufiger als zweimal pro Woche in einem Monat sowie spontaner Remission

- Halluzinationen

- Wahn

- Formale Denkstörungen

Vorliegen eines Risikofaktors (familiäre Belastung mit einer schizophrenen Spektrumstörung oder schizotype Persönlichkeitsstörung beim Patienten) und einem Absinken des globalen Funktionsniveaus (Abfall im GAF-Gesamtwert um mindestens 30\%). 
Abb. 1 Frühverlauf der Schizophrenie

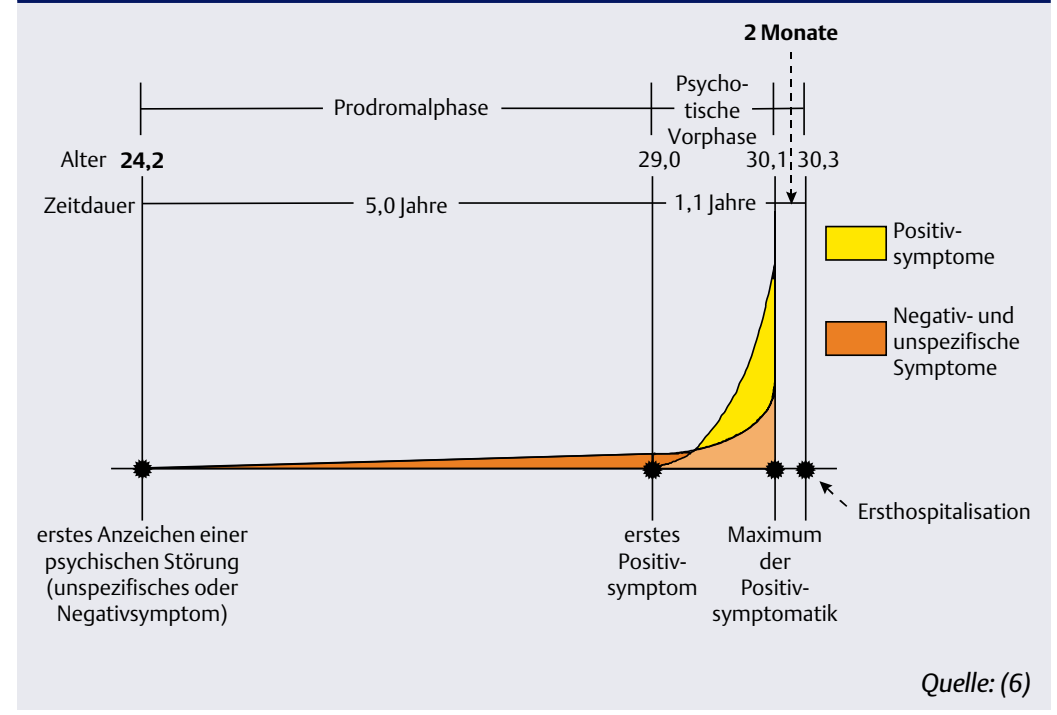

mes bzw. das Structured Interview for Prodromal Syndromes (SOPS/SIPS; 15), die Schizophrenia Prediction Scale, Adult version, SPI-A (20) und das Early Recognition Instrument based on the Instrument for the Retrospective Assessment of the Onset of Schizophrenia, ERIraos (12). Während die CAARMS, die SOPS/SIPS und das ERIraos auf die Erfassung einer breiten Symptomatik abzielen, ist die SPIA speziell für die Erfassung von Basissymptomen in ihrer quantitativen Ausprägung und damit als eine kom-

\section{Abb. 2 Reduktion von grauer Substanz}

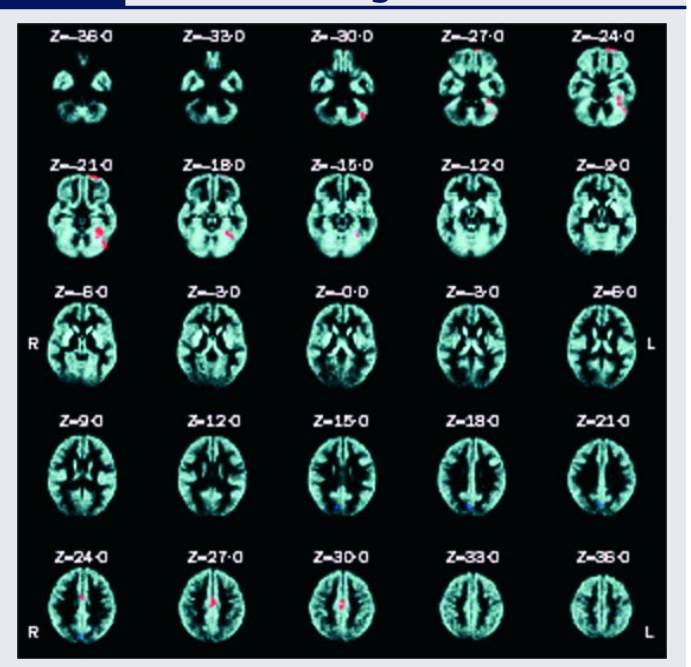

Reduktion von grauer Substanz vor allem parahippokampal, fusiform, orbitofrontal, zerebellär und im Cingulum (MRT, rote Markierung) vor und nach Erstmanifestation einer schizophrenen Psychose bei 12 Patienten (Quelle: (18)) plementäre Skala zur SOPS/SIPS konzipiert worden. Da die Erhebung dieser Instrumente relativ viel Zeit in Anspruch nimmt und ihr Einsatz unter routinemäßigen ambulanten Versorgungsbedingungen nur ausnahmsweise geleistet werden kann, bestehen zusätzlich Bestrebungen, praktikablere Screeningverfahren zu entwickeln. Im Rahmen des BMBF-geförderten Kompetenznetzes Schizophrenie wurde darum eine aus 17 Fragen bestehende sogenannte „Checkliste“ entwickelt, deren Durchführung nicht Sie ist bewusst weit gefasst, um mögliche Risikopersonen nicht vorschnell auszuschließen. Bei Erreichen bzw. Überschreiten eines festgelegten Cutoffs, d.h. dem möglichen Vorliegen eines Risikos, kommen dann als zweite Stufe in einem spezialisierten Früherkennungszentrum die ausführlichen Früherkennungsinstrumente zum Einsatz.

Unterstützt wird diese klinische Einschätzung, die sich in der Regel über mehrere Termine erstreckt, durch Untersuchungen, wie man sie bei jeder „Erstmanifestation“ durchführen würde: Erfassung möglicher Risikofaktoren, allgemeiner psychopathologischer Befund, körperliche Untersuchung, Labor, neuropsychologische Testung, EEG, Bildgebung. Da die ersten Zeichen einer beginnenden Schizophrenie meist schon in der Pubertät auftreten, ist der enge Kontakt zu Kinder- und Jugendpsymehr als zehn Minuten beansprucht. chiatern sehr zu empfehlen, wie es beispielsweise die Früherkennungszentren in Berlin-Brandenburg, Heidelberg und neuerdings in Hamburg institutionalisiert haben. Entsprechende Ratingskalen für die sich bei sehr jungen Patienten z.T. etwas anders akzentuierende Prodromalsymptomatik sind im Entstehen.

Eine erfolgreiche Früherkennung setzt eine breite Aufklärung der allgemeinen und fachlichen Öffentlichkeit und deren Sensibilisierung für das Thema der Schizophrenie und ihres Frühverlaufs voraus. Hierzu bedarf es eines direkten Kontaktes zu möglicherweise betroffenen Menschen oder deren Angehörigen bzw. zu sogenannten Multiplikatoren, d.h. vermittelnden Personen, die durch ihre berufliche oder institutionelle Stellung direkten Kontakt mit den betroffenen Personen im jugendlichen und jungen Erwachsenenalter haben. Dabei kann es sich um Ärzte und Psychologen handeln, aber auch um Personen, die in komplementären Einrichtungen tätig sind (Beratungsstellen, Betreuungseinrichtungen, Lehrer, Seelsorger, Bundeswehr, etc.). In Berlin-Brandenburg ist beispielsweise gegenwärtig, ähnlich der Aktivitäten des Kölner Früherkennungsund Therapiezentrums für psychische Krisen (FETZ) als der ersten Einrichtung dieser Art in Deutschland, ein breit angelegtes lokales Netzwerk für Früherkennung von Prodromalzuständen psychotischer Erkrankungen im Entstehen. Eine ganze Reihe von Vorfeldeinrichtungen wie z.B. andere psychiatrische Krankenhäuser, Praxisnetze und psychosoziale Beratungseinrichtungen arbeiten bereits in diesem Netzwerk mit. Des weiteren wurde eine Vielzahl von Fortbildungs- und Informationsveranstaltungen für Lehrer, Nervenärzte, Sozialarbeiter, Psychologen etc. durchgeführt. Durch solche Maßnahmen konnten die Bekanntheit des Themas in der Region und die Zahl der Kontakt suchenden Patienten und der dann erkannten Prodromalpatienten deutlich erhöht werden.

Früherkennung führt dann zur Frühintervention oder Frühbehandlung (13). Hierzu werden gegenwärtig in Deutschland und international verschiedene Studien durchgeführt. Ers- 
te Ergebnisse weisen darauf hin, dass der Einsatz niedrig dosierter atypischer Neuroleptika bei Patienten mit APS oder BLIPS zu einer Besserung der Symptomatik sowie zu einer Reduktion der Übergangswahrscheinlichkeit zumindest während des Behandlungszeitraumes führt $(14,19,21)$. Auch kognitiv-behaviorale Ansätze scheinen sowohl in dieser späten als auch in früheren Phasen günstige Effekte zu zeitigen $(2,16)$. Es ist aber noch deutlich zu früh, hieraus bereits allgemeine Behandlungsempfehlungen abzuleiten, so dass Interventionen in der Prodromalphase einstweilen nur im Rahmen kontrollierter wissenschaftlicher Studien in entsprechend spezialisierten Zentren stattfinden können. Fernziel all dieser Bemühungen in der Prodromalphase ist es, den Ausbruch des Vollbildes der Psychose zu verhindern, bzw. abzumildern und damit insgesamt die Prognose und den Langzeitverlauf schizophrener Erkrankungen sozial und neurobiologisch zu verbessern.

\section{Early Detection of Incipient Schizophrenic Diseases} Schizophrenic disorders continue to have a poor prognosis. Chronic courses with appreciably social deficits and progressive neurobiological changes are frequent. It is today known that the longer the untreated period prior to initial hospitalisation, the poorer the subsequent prognosis is going to be. Strategies of early recognition of patients in the prodromal phase with the aim of timely treatment, therefore make good sense. For the recognition in particular of early prodromal stages, the Bonn-Cologne based symptom concept, and especially a subgroup of cognitive-perceptive basic symptoms, has proven to be specific for and predictive of the probability of transition to the full picture of a schizophrenic psychosis. In the later phases of the prodrome, in which transition to a manifest psychosis is already imminent, the socalled attenuated psychotic symptoms (APS) and brief limited intermittent psychotic symptoms (BLIPS) - criteria being close to that of a schizotypical personality disorder - are applied. A whole range of structured interviews and rating scales (e.g. SIPS, SPIA, ERI- raos) have been developed to standardize the early recognition of schizophrenic prodromal states. These are embedded in a series of further examinations comprising neuropsychological, neurophysiological and neuroimaging techniques. For successful early recognition, however, the awareness of the issue of schizophrenia and its early course needs to be widely promoted among both the public at large and the relevant professionals. The establishment of networks between specialized early recognition and intervention centres and as many professional groups working with adolescents and young adults as possible, would appear to be of particular importance.

\section{Literatur}

1. Andreasen NC, Flaum M. Schizophrenia: the characteristic symptoms. Schizophr Bull 1991;17(1): 27-49

2. Bechdolf A, Maier S, Knost B et al. Psychologisches Frühinterventionsprogramm für psychosefernen Prodromen - Ein Fallbericht. Nervenarzt 2003; 74: 436-439

3. Gschwandtner U, Aston J, Borgwardt S et al. Basel Early Detection of Psychosis Study - Früherkennung von Psychosen (FEPSY). Neuropsychological and neurophysiological findings in individuals suspected to be at risk for schizophrenia: preliminary results from the Basel early detection of psychosis study - Früherkennung von Psychosen (FEPSY). Acta Psychiatr Scand 2003; 108: 152-155

4. Gouzoulis-Mayfrank E. Komorbidität Psychose und Sucht. Von den Grundlagen zur Praxis. Darmstadt, Steinkopf, 2003

5. Häfner H, Maurer K, Loffler W, RiecherRossler $A$. The influence of age and sex on the onset and early course of schizophrenia. BrJ Psychiatry 1993; 162: 80-86

6. Häfner H, Maurer K, Löffler W et al. Onset and early course of schizophrenia. In: Häfner H, Gattaz H (eds) Search for the Causes of Schizophrenia, Voll III. Berlin, Springer-Verlag, 1995, 43-66

7. Huber G. Reine Defektsyndrome und Basisstadien enodgener Psychosen. Fortschr Neurol Psychiatr 1966; 34: 409-26

8. Huber $\mathrm{G}$. The heterogeneous course of schizophrenia. Schizophr Res 1997; 28: 177-185

9. Jackson HJ, McGorry PD, Dakis J et al. The inter-rater and test-retest reliabilities of prodromal symptoms in first-episode psychosis. Aust N Z J Psychiatry 1996; 30 : 498-504

10. Klosterkötter J, Hellmich M, Steinmeyer EM, Schultze-Lutter F. Diagnosing schizophrenia in the initial prodromal phase. Arch Gen Psychiatry 2001; 58: 158-64

11. Larsen TK, McGlashan TH, Johannessen JO et al. Shortened duration of untreated first episode of psychosis: changes in patient characteristics at treatment. Am J Psychiatry
2001; 158: 1917-1919

12. Maurer K, Hörrmann F, Schmidt M et al. The early recognition inventory: structure, reliability and initial results. Schizophr Res 2004; 67 (suppl): 34

13. McGlashan TH, Miller TJ, Woods SW. Pre-onset detection and intervention research in schizophrenia psychoses: current estimates of benefit and risk. Schizophr Bull 2001; 27: 563-570

14. McGorry PD, Yung AR, Phillips LJ et al. Randomized controlled trial of interventions designed to reduce the risk of progression to first-episode psychosis in a clinical sample with subthreshold symptoms. Arch Gen Psychiatry 2002; 59: 921-928

15. Miller TJ, McGlashan TH, Woods SW et al. Symptom assessment in schizophrenic prodromal states. Psychiatr Q 1999; 70: 273-287

16. Morrison AP, Bentall RP, French P et al. Randomised controlled trial of early detection and cognitive therapy for preventing transition to psychosis in high-risk individuals. Study design and interim analysis of transition rate and psychological risk factors. Br] Psychiatry 2002; Suppl 43: S78-84 17. Murray CJ, Lopez AD. Global mortality, disability, and the contribution of risk factors: Global Burden of Disease Study. Lancet 1997; 349: 1436-42

18. Pantelis C, Velakoulis D, McGorry PD et al. Neuroanatomical abnormalities before and after onset of psychosis: a cross-sectional and longitudinal MRI comparison. Lancet $2003 ; 361: 281-288$

19. Ruhrmann S, Kühn KIJ, Streit M et al. Pharmakologische und psychologische Frühintervention bei Risikopersonen mit psychosenahen Prodromen: Erste Ergebnisse einer kontrollierten Studie. Nervenarzt 2002; 73 (Supp. 1): 9

20. Schultze-Lutter $F$, Steinmeyer EM, Picker $\mathrm{H}$ et al. Detecting schizophrenia in the early prodrome. Schizophr Res 2004; 67 (suppl): 19-20

21. Woods SW, Breier A, Zipursky RB et al. Randomized trial of olanzapine versus placebo in the symptomatic acute treatment of the schizophrenic prodrome. Biol Psychiatry 2003; 54: 453-64.

22. Yung AR, McGorry PD. The initial prodrome in psychosis: descriptive and qualitative aspects. Aust N Z J Psychiatry 1996; 30 : 587-599

23. Yung AR, Phillips LJ, McGorry PD et al. Prediction of psychosis. A step towards indicated prevention of schizophrenia. Br J Psychiatry Suppl 1998; 172: 14-20

24. Yung AR, Phillips LJ, Yuen HP et al. Psychosis prediction: 12-month follow up of a high-risk („,prodromal“) group. Schizophr Res 2003; 60: 21-32

\section{Korrespondenzadresse}

PD Dr. med. Georg Juckel

Früherkennungs- und Therapiezentrum

für beginnende Psychosen Berlin-Brandenburg, Klinik für Psychiatrie und Psychotherapie, Charité, Campus Mitte

Schumannstr. 20/21

10117 Berlin

georg.juckel@charite.de 